Voix et Images

voixetimages

\title{
La psychanalyse ou comment s'en débarrasser
}

\section{Anne Élaine Cliche}

Volume 15, numéro 1 (43), automne 1989

Jacques Poulin

URI : https://id.erudit.org/iderudit/200822ar

DOI : https://doi.org/10.7202/200822ar

Aller au sommaire du numéro

\section{Éditeur(s)}

Université du Québec à Montréal

\section{ISSN}

0318-9201 (imprimé)

1705-933X (numérique)

Découvrir la revue

\section{Citer cet article}

Cliche, A. É. (1989). La psychanalyse ou comment s'en débarrasser. Voix et Images, 15(1), 117-120. https://doi.org/10.7202/200822ar d'utilisation que vous pouvez consulter en ligne.

https://apropos.erudit.org/fr/usagers/politique-dutilisation/ 


\section{La psychanalyse ou comment s'en débarrasser}

\section{par Anne Élaine Cliche, Université d'Ottawa}

Il s'agit, je crois, d'un malentendu. À moins que ce ne soit, encore plus simplement, l'effet d'une certaine surdité dont on fait depuis longtemps ici - je veux dire au Québec - une pratique courante. Quoi qu'il en soit, Du rêve au texte de Henri-Paul Jacques se présente avant tout comme l'assemblage de quelques articles et travaux (surtout des cours) étalés sur une quinzaine d'années et dont la visée s'affiche en page couverture à la fois comme un objectif et un parti pris pour une narratologie et une poétique psychanalytiques 1 .

La question consiste d'abord à se demander quel peut bien être le statut de la psychanalyse lorsqu'elle est ainsi ramenée, voire confinée à une fonction prédicative. En quoi une narratologie et une poétique sont-elles appelées à «être» psychanalytiques? Ou encore: qu'advient-il du psychanalytique lorsque appliqué ainsi aux soi-disant sciences du texte? Questions difficiles sinon déterminantes auxquelles le titre de ce recueil d'essais a au moins l'avantage d'offrir l'élément d'une réponse toute faite: celui qui consisterait à «faire passer» le travail de l'inconscient à l'écrit. Or, de la première à la dernière page, il s'agit bien de démontrer et de redémontrer l'adéquation du rêve - entendu ici comme processus psychique relevant de la condensation et du déplacement - au texte - entendu, lui, comme le résultat tangible d'un transfert (conscient ou non) desdits processus. Qu'est-ce à dire? Premièrement, que le texte sera toujours cet objet chu (sinon déchu), concret et manipulable en tant qu'effet d'une causalité psychique pratiquement «référentielle». Ensuite, que le travail du rêve devient ici une «méthode» ou, de l'aveu même de l'auteur, une «grille» dont l'utilisation consiste à nous détourner du texte pour faire de celui-ci le trait probant d'un auteur quand ce n'est des catégories freudiennes elles-mêmes.

Dès lors, dans un tel passage du rêve au texte, qu'est-ce qui se perd? Rien moins que le sujet, c'est-à-dire l'effet-coupure ou l'effet-signifiant de l'écriture. Et qu'est-ce qui revient comme par miracle au premier plan des préoccupations? L'auteur dans son versant le plus éculé de producteur de fantasme dont la fonction centrale se résume à faire de la langue le reflet objectal de celui-ci. De là, l'Inconscient (majuscule) peut se confondre avec le Pré-conscient ${ }^{2}$ ou pire, s'apparenter à un code (p. 45) dont l'absolu dictionnaire (??) ne nous renvoie jamais qu'à l'intentionnalité - probable/improbable - de celui qui écrit. D'ailleurs ici, qui rêve et qui écrit ne font qu'un seul être puisqu'on nous dit sans rire que, par exemple, la narratrice [est] identifiable à Anne Hébert (p. 48), ou encore que la lecture consiste à remonter de l'être-de-papier à son créateur comme «Sujet» ou «écrivain-Sujet» (p. 266, 143).

Le malentendu est tellement énorme, tellement courant, qu'on se décourage d'avoir à le pointer du doigt. Quel est donc le sujet de la psychanalyse? Le sujet de l'inconscient, bien sûr, mais de quel inconscient? Certainement pas d'un 
inconscient-dictionnaire-absolu (p. 63, 70) ni d'un inconscient-code. Ces derniers ne nous conduisent jamais qu'à la psychè ou à une sorte de substrat de l'identité permanente - ici le texte comme substrat de l'individu écrivain. Lacan - car c'est AUSSI de lui qu'il faudrait parler - n'a cessé de l'affirmer sous plusieurs formes: Le sujet sur quoi nous opérons en psychanalyse ne peut être que le sujet de la science. ${ }^{3} L^{\prime}$ inconscient $n^{\prime}$ est pas le rêve, dit, pour sa part, Freud. Disons-le brutalement: il n'y a pas de représentation possible de l'inconscient du sujet, écrivain ou non. Un signifiant ne représente un sujet que POUR un autre signifiant, appelé mais à jamais absent. Il n'y a pas d'autre définition du signifiant que celle-là qui se fonde sur un impossible logique. Or, c'est ce manque irréductible entre deux signifiants qui est cause du sujet. Non pas selon l'ordre d'une antécédence causale mais dans l'après-coup de la certitude de penser (cogito cartésien inversé puisque je suis est l'effet du je pense). Le sujet de la science est un sujet frappé d'un impératif qui le presse d'assumer sa propre causalité (Lacan). Wo es war soll Ich werden (Freud). Autrement dit, il n'y a de sujet que de la certitude d'être né de cette béance où je reste innommé.

La scansion lacanienne, comme dit Henri-Paul Jacques (p. 264), n'est pas tant lacanienne que réelle en ce qu'elle est cette béance insistante faisant advenir une vérité qui n'est pas de l'ordre du savoir, du représentable ou de l'expression. Couper un mot dans un texte pour figurer ce qui s'y dit de rupture ${ }^{4}$ n'a pas d'autre sens que celui de chercher à rendre au visible et au représenté ce qui y fait bord. Procédé qui prend le mot pour la chose et s'y complait. Ajoutons, en revenant au travail de Jacques, que l'inconscient freudien n'est pas réductible au refoulé (l'idée du dictionnaire absolu vient de la croyance en un inconsciententrepôt des images, figures ou mots refoulés). L'inconscient, pour reprendre un jeu de mot de Lacan sur le terme allemand unbewusst, c'est l'unebévue, c'est-àdire ce qui achoppe, dérape ou rate dans l'élaboration qu'est, par exemple, la suite des pensées du rêve. Et c'est là que doit prendre place l'interprétation. Qu'est-ce qui manque et manquera toujours à sa place? Un signifiant pour dire qui je suis. L'interprétation consistera donc à couper dans la parole pour que la FORME de ce manque s'y fasse enfin entendre.

À cela, Henri-Paul Jacques (et bien d'autres avec lui) reste sourd. Chacune de ses conclusions est la reprise d'une formule tautologique qui pourrait s'énoncer ainsi: le texte représente l'auteur parce que l'auteur c'est son texte.

Comme si l'écrivain-Sujet pouvait exceptionnellement échapper à la cohérence humaine profonde qui se trouve être notre lot à tous même à travers nos incohérences superficielles apparentes. (p. 143-144)

Que conclure [...]? À une seule chose. Au retour progressif d'un refoulé qui véhicule les traces anciennes d'une scène perçue (ou fantasmée?) [par Anne Hébert] durant l'enfance et dite "primitive» ou «originaire». (p. 49-50)

Et voilà pour la psychocritique ravalée à son rang d'usage et d'application. D'ailleurs l'incipit du livre était clair: Application de la psychanalyse à la 
littérature (p. 21). Nous sommes fixés, et pour de bon. Mais au fond, qu'en estil des soi-disant rapports entre la psychanalyse et la littérature? Avant toute argumentation, j'aimerais oser répondre à Henri-Paul Jacques que si l'écrivainSujet n'échappe pas à la cohérence humaine, le sujet écrivain - ou sujet de l'écriture -, lui, y échappe. Il en va même de son nom, voire de son re-nom, autant dire de sa signature.

Si Freud est allé aux limites refoulées du sens commun, s'il a exhumé l'horreur du mal sur lequel se fonde l'humanité (et dont Jean Bellemin-Noël s'empresse de nous rappeler la portée en préface des essais de Jacques), on ne doit pas oublier la résistance du même Freud à l'irréductibilité des textes littéraires qu'il a toujours pris pour matériaux d'analyse au service des structurations psychanalytiques (voir ses lectures de Dostoïevski, Jensen, Hoffmann). C'est Lacan, contemporain de Joyce, qui nous a rappelés à une pensée du texte directement à l'œuvre dans la lecture comme acte d'un sujet de langage, c'est-àdire de désir. Pour Freud, l'écrivain est un homme (raison classique et suffisante) et son texte ne peut que nous ramener à l'humain. Après Lacan, après ce qu'il nous a transmis de la logique du signifiant en tant qu'elle engage tout acte de langage, l'écrivain n'est plus que le sujet qui, parcourant le chemin de sa nomination pour le porter à l'écrit, expose le procès même qui l'a constitué et ce faisant, donne forme à ce qui, manquant à son nom, l'a nommé. L'inhumanité d'un tel sujet fait signature.

Pendant ce temps, Henri-Paul Jacques consacre presque tout son recueil aux figures de rhétorique prises dans leur versant imitatif. Selon les termes de l'essayiste, il s'agirait d'un retour de l'exprimé dans l'expression, à moins que ce ne soit le contraire puisque d'un côté comme de l'autre, il s'agit de débusquer la représentation matérielle spatiale de l'idée exprimée (p. 230) selon une pratique ouvertement pavlovienne ${ }^{5}$ qui consiste à faire - le plus doctement du monde - de l'idée ou de l'image exprimée, la cause (au sens de stimulus) de l'expression (au sens de réaction).

S'il fallait partir de Freud et contourner Lacan pour en arriver là, on est en droit de se demander en quoi l'entrée de la psychanalyse à l'université - car c'est bien de cela qu'il est question - peut nous apporter quelque chose. Un tel recours à la causalité (dans sa forme la plus horriblement simpliste) s'accompagne, on s'y attendait, d'un anti-intellectualisme diffus mais insistant dans tous les recoins du livre.

$\mathrm{Ne}$ nous emballons pas. Disons seulement que si la psychanalyse nous amène directement au roman familial et au sujet de l'écriture, ce n'est certainement pas pour revenir à la notion transcendante d'auteur et à tous les lieux communs qui en découlent. La psychanalyse n'est pas une doctrine (malgré ce qu'en pense Jean Bellemin-Noël), ni une grille. Elle est radicalement impropre à l'usage universitaire puisqu'elle est d'abord une pensée de l'inconscient (et non une pensée SUR l'inconscient) qui force celui qui s'y prête à reconnaitre la place qu'il occupe dans la langue en tant que sujet de désir. La psychanalyse ne s'installe qu'en exil du savoir, sur une autre scène que les sciences dites «humaines», parce que sa vérité est celle d'une absence au champ du savoir lui- 
même. Est-ce dire que le savoir en est exclu? Certainement non. Mais il borde ce qui s'y dérobe et qui est le sujet en question. La psychanalyse à l'université n'est nécessaire que si elle ouvre la pensée universitaire - encore faut-il qu'il y en ait une - à ce qui lui est radicalement hétérogène. La psychanalyse ne répond pas aux impératifs des institutions d'enseignement sauf à abolir sans reste son acte analytique. Sa transmission est de l'ordre d'une extériorité interne. Autant dire qu'elle ne peut être «appliquée» que si l'on souhaite irrémédiablement s'en đébarasser.

Ainsi est inaugurée la nouvelle collection «Études André Belleau» aux éditions Guérin littérature. Collection dont les critères d'excellence nous sont détaillés au dos de la couverture ainsi que dans la «Présentation» qui ouvre le livre: qualités intrinsèques des recherches, traitement achevé des sujets, originalité et excellence des travaux produits. Il y a là, me semble-t-il, un symptôme. La reconnaissance ostentatoire par l'institution des lettres (chapeautée d'une préface et d'un hommage dont le kitsch du signataire n'a d'égal que 1'insistance avec laquelle il nie la place inassimilable de la psychanalyse 6 ), cette reconnaissance, donc, d'un ouvrage ouvertement psychanalytique et qui n'est en réalité qu'un usage de ce que l'auteur appelle lui-même les étiquettes freudiennes, ne vient-elle pas révéler une fois de plus la résistance solide des intellectuels d'ici à un nom propre dont nul ne s'attache vraiment à creuser les effets: Lacan. Il faudra dire et redire sous plusieurs formes en quoi et pourquoi ce nom est aujourd'hui incontournable.

1 Henri-Paul Jacques, Du rêve au texte. Pour une narratologie et une poétique psychanalytiques, Montréal, Université du Québec à Montréal/Guérin littérature, 1988,348 p. (Etudes André Belleau).

$2 Q u^{\prime}$ on ne cherche pas dans ce qui suit des distinctions métapsychologiques rigoureuses entre Inconscient et Préconscient. J'ai voulu garder aux étiquettes freudiennes leur souplesse originelle [...]. (p. 29)

3 Jacques Lacan, Écrits, Paris, Seuil, 1966, p. 858.

4 Henri-Paul Jacques consacre l'essentiel de son livre à l'étude de certaines figures de rhétorique dont la caractéristique commune consiste à rompre et à réaménager le matériau linguistique (antanaclase, calligramme).

5 Du point de vue du narrateur-auteur et dans le cadre du phénomène associatif, ces deux types d' influenceldéterminisme de l'idée obsédante peuvent se conceptualiser assez simplement à partir du modèle pavlovien repris par le behaviorisme, «stimulus $\rightarrow$ réaction»; ou pour conserver la terminologie traditionnelle consacrée, "mot-inducteur (pensée inductrice) $\rightarrow$ mot induit (pensée induite)». [...] Le stimulus se (re)constitue d' un élément textuel. (p. 86-87)

6 Jean Bellemin-Noël signe l'hommage au défunt auteur et la préface. Pour le kitsch: Henri-Paul Jacques nous a quittés le vendredi 7 octobre. [...] Bonne randonnée camarade! En guise de mouchoirs, pour saluer ton départ, nous agitons dans le ciel d'automne ces pages dont la voix à jamais tremble de vie. (p. 14) Pour la psychanalyse: On accordera de surcroît que la doctrine inventée par Freud $[. .$.$] a$ du mal à démêler, et d'abord à définir, ses vérités [...]. On a beau avoir été - 'professeur durant des lustres, quand on s'occupe de psychanalyse on est moins que d'autres assuré de détenir quelque maîtrise. [...] [l']auteur a eu l'heureuse idée de reconnaître et de nous prouver qu'il est, fût-ce malgré lui, un maître. (p. 12-13) Cette demière phrase n'est d'ailleurs pas sans ambiguitté. 